\title{
EFFECTS OF AN AXISYMMETRIC RIGID PUNCH ON A NONHOMOGENEOUS TRANSVERSELY ISOTROPIC HALF-SPACE
}

\author{
P. K. CHAUDHURI ${ }^{1}$ and SUBHANKAR RAY ${ }^{1}$
}

(Received 15 December, 1999; revised 4 July, 2001)

\begin{abstract}
Elastic behaviour of a nonhomogeneous transversely isotropic half-space is studied under the action of a smooth rigid axisymmetric indentor. Hankel transforms of different orders have been used. It is observed that in contrast to a homogeneous medium, the pressure distribution in the contact region in a nonhomogeneous medium is not directly available, rather it is obtainable from the solution of a Fredholm integral equation. The integral equation is solved for a flat-ended punch and paraboloidal indentations for various values of the nonhomogeneity parameter, and the effects of nonhomogeneity in elastic behaviour on stresses have been shown graphically. The results of the associated homogeneous case are readily available from the results of the present study.
\end{abstract}

\section{Introduction}

The determination of the elastic displacement field in the half-space under the action of a rigid indentor has been the subject of much interest and various methods have been employed for the solution of such problems: for example, Spence $[10,11]$ applied the Wiener-Hopf method, and Sneddon [9] obtained a relation between load and penetration for a punch of arbitrary profile using the integral transform method. A systematic description of such works has been given by Gladwell [3]. A solution of the axisymmetric Boussinesq problem for an initially stressed Neo-Hookean halfspace has been reported by Dhaliwal and Singh [2]. Ting [12] considered contact stresses between a rigid indentor and a viscoelastic half-space. Most of the works in this field have been limited to isotropic elastic media. But increased use of anisotropic materials in engineering applications has resulted in considerable attention being focused on media of anisotropic character. Some works in this field are available in Gladwell's book [3]. One basic problem that arises in considering an anisotropic

\footnotetext{
'Department of Applied Mathematics, University College of Science, 92 A.P.C.Road, Calcutta 700009 , India; e-mail: pkcha@vsnl.net.

(C) Australian Mathematical Society 2003, Serial-fee code 1446-1811/03
} 
medium is that, except for some particular types of anisotropy it is not possible to write displacement components in terms of potential functions in a general anisotropic medium (Lekhnitskii [6]). Stress distribution in an anisotropic elastic half-space under axially symmetric normal load has been discussed by Lekhnitskii [6]. More recently, the strain fields in a transversely isotropic half-space have been investigated by Pouyet and Lataillade [8], who used Hankel transforms of different orders to solve the problem.

Positional variation of elastic coefficients leads to a nonhomogeneous elastic medium, which is a very important concept from a practical point of view. But this area has not been studied rigorously possibly due to the severe difficulty in handling the governing differential equations for such a medium. Sometimes it is observed that even for a comparatively straightforward case of power law variations, the governing equations appear too difficult. It is possible that this is why investigations in this area have thus far been limited to special types of variations in elastic coefficients (see, for example, $[1,5,7])$, rather than consideration of general variations.

The present study focuses on the determination of elastic strains, penetration depth and pressure distribution etc. in a nonhomogeneous transversely isotropic half-space caused by a rigid punch. Nonhomogeneity in elastic coefficients $c_{i j}$ has been assumed in the form $c_{i j}=c_{i j}^{0} e^{\alpha z}, \alpha$ being a real constant. As in [8], Hankel transforms of different orders have been applied to find the solution. In contrast to the homogeneous case, the equation for pressure distribution is a Fredholm integral equation of the second kind. The integral equation has been solved numerically and the stresses have been computed. The effects of nonhomogeneity on stresses have been represented graphically for some special cases of indentation. Finally, all the results for the homogeneous medium may be obtained from the results of the present discussion by putting $\alpha=0$.

\section{The basic equations}

We consider an elastic half-space of transversely isotropic material and suppose that the plane of isotropy passing through each point of the body is parallel to the plane boundary of the half-space. Taking the $z$-axis along the normal to the half-space boundary and pointing inwards and using cylindrical coordinates $(r, \theta, z)$, the stressstrain relations are given by $\left[\sigma_{r}, \sigma_{\theta}, \sigma_{z}, \sigma_{r z}\right]^{T}=\left[C^{*}\right]\left[\varepsilon_{r}, \varepsilon_{\theta}, \varepsilon_{z}, \varepsilon_{r z}\right]^{T}$, where $\left[C^{*}\right]$ is the elastic coefficient matrix such that

$$
\left[C^{*}\right]=\left(\begin{array}{cccc}
c_{11} & c_{12} & c_{13} & 0 \\
c_{12} & c_{11} & c_{13} & 0 \\
c_{13} & c_{13} & c_{33} & 0 \\
0 & 0 & 0 & c_{44}
\end{array}\right)
$$


Because of elastic symmetry and the symmetry of the force distribution the displacement components are independent of $\theta$ and we may take

$$
u_{r}=u(r, z), \quad u_{\theta}=0, \quad u_{z}=w(r, z)
$$

and the strain components related to the displacement components given by (2.1) are

$$
\varepsilon_{r}=\frac{\partial u}{\partial r}, \quad \varepsilon_{\theta}=\frac{u}{r}, \quad \varepsilon_{z}=\frac{\partial w}{\partial z}, \quad \varepsilon_{r z}=\frac{\partial u}{\partial z}+\frac{\partial w}{\partial r}, \quad \varepsilon_{r \theta}=\varepsilon_{\theta z}=0 .
$$

The equations of equilibrium are

$$
\frac{\partial \sigma_{r}}{\partial r}+\frac{1}{r}\left(\sigma_{r}-\sigma_{\theta}\right)+\frac{\partial \sigma_{r z}}{\partial z}=0, \quad \frac{\partial \sigma_{r z}}{\partial r}+\frac{\sigma_{r z}}{r}+\frac{\partial \sigma_{z}}{\partial z}=0 .
$$

We assume that the nonhomogeneity of the elastic medium is due to the dependence of the $c_{i j}$ 's on depth, in the form $c_{i j}=c_{i j}^{0} e^{\alpha z}$, where $c_{i j}^{0}$ and $\alpha$ are constants.

\section{Boundary conditions}

Let the tip of the axisymmetric as well as smooth punch penetrate through a depth $D$ (at present unknown) into the half-space. Taking the tip of the punch as the origin, let $z=f(r)$ be the equation of the punch, with the condition that $f(0)=0$. The function $f(r)$ together with its first derivative is assumed to be continuous, except possibly on the axis.

The boundary conditions for the problem are as follows:

$$
\begin{aligned}
& \sigma_{z}(r, 0)=0, \quad r>a, \\
& \sigma_{r z}(r, 0)=0, \quad \forall r
\end{aligned}
$$

and

$$
D-f(r)=w(r, 0) .
$$

Here we assume that $a$ is the radius of the circle of contact.

In addition to the above boundary conditions we must have the obvious regularity conditions that the stresses and displacements should tend to zero as $\sqrt{r^{2}+z^{2}} \rightarrow \infty$.

\section{Use of Hankel transforms}

Following [8], we use Hankel transforms of different orders with respect to the variable $r$ to express the basic equations and boundary conditions in terms of the single variable $z$. We shall denote the Hankel transform of order $v$ of a function $f(r)$ by $\bar{f}(q)$ such that $\bar{f}(q)=\int_{0}^{\infty} r f(r) J_{\nu}(q r) d r$.

In our subsequent analysis we shall apply a Hankel transform of 


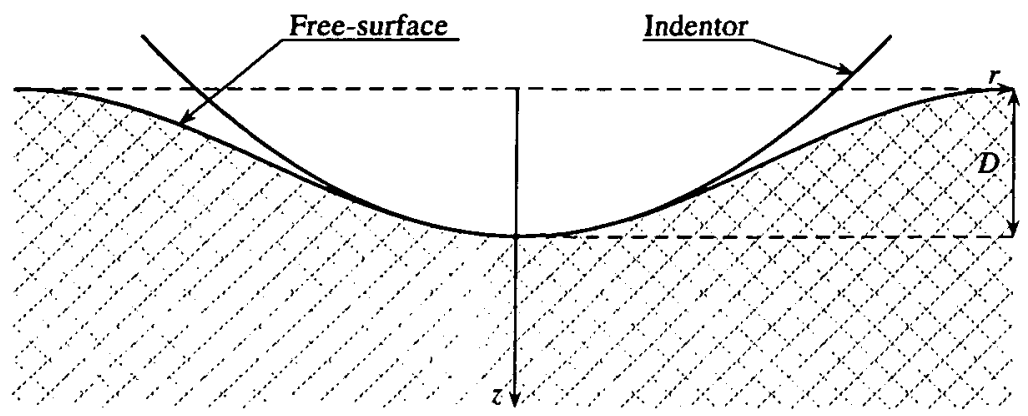

FIGURE 1. Rigid indentor on a half-space.

(i) zero order to $\sigma_{r}+\sigma_{\theta}, \sigma_{z}, \varepsilon_{r}+\varepsilon_{\theta}, \varepsilon_{z}$ and $w$;

(ii) order one to $\sigma_{r z}, \varepsilon_{r z}$ and $u$;

(iii) order two to $\sigma_{r}-\sigma_{\theta}, \varepsilon_{r}-\varepsilon_{\theta}$.

Then in terms of the transformed functions the equations of equilibrium (2.3) become

$$
q\left(\overline{\sigma_{r}-\sigma_{\theta}}\right)-q\left(\overline{\sigma_{r}+\sigma_{\theta}}\right)+2 \frac{d \bar{\sigma}_{r z}}{d z}=0, \quad q \bar{\sigma}_{r z}+\frac{d \bar{\sigma}_{z}}{d z}=0 .
$$

The stress-strain relations become

$$
\begin{aligned}
\overline{\sigma_{r}+\sigma_{\theta}} & =c_{44}\left[q\left(a_{3}+a_{4}\right) \bar{u}+2 a_{1} \frac{d \bar{w}}{d z}\right], & \bar{\sigma}_{z} & =c_{44}\left[q a_{1} \bar{u}+a_{2} \frac{d \bar{w}}{d z}\right], \\
\overline{\sigma_{r}-\sigma_{\theta}} & =c_{44} q\left(a_{4}-a_{3}\right) \bar{u}, & \bar{\sigma}_{r z} & =c_{44}\left[\frac{d \bar{u}}{d z}-q \bar{w}\right],
\end{aligned}
$$

where

$$
\begin{gathered}
a_{1}=c_{13}^{0} / c_{44}^{0}, \quad a_{2}=c_{33}^{0} / c_{44}^{0}, \quad a_{3}=c_{11}^{0} / c_{44}^{0}, \quad a_{4}=c_{12}^{0} / c_{44}^{0} . \\
\overline{\varepsilon_{r}+\varepsilon_{\theta}}=q \bar{u}, \quad \overline{\varepsilon_{r}-\varepsilon_{\theta}}=-q \bar{u}, \quad \bar{\varepsilon}_{z}=\frac{d \bar{w}}{d z}, \quad \bar{\varepsilon}_{r z}=\frac{d \bar{u}}{d z}-q \bar{w} .
\end{gathered}
$$

If $p(r)$ is the pressure of the punch on $z=0$, then

$$
\sigma_{z}(r, 0)=-p(r), \quad r \leq a .
$$

Hankel transforms of boundary condition (3.1) and Equation (4.4) yield

$$
\bar{\sigma}_{r z}(q, 0)=0 \text { and } \bar{\sigma}_{z}(q, 0)=-\bar{p}(q)
$$

where

$$
\bar{p}(q)=\int_{0}^{a} r p(r) J_{0}(q r) d r
$$




\section{Method of solution}

It can easily be shown by substitution of (4.2) into (4.1) that $\bar{u}$ and $\bar{w}$ satisfy the following linear differential equation in $z$ :

$$
\left(\frac{d^{2}}{d z^{2}}+\alpha \frac{d}{d z}-q^{2} \delta_{1}^{2}\right)\left(\frac{d^{2}}{d z^{2}}+\alpha \frac{d}{d z}-q^{2} \delta_{2}^{2}\right)[\bar{u}, \bar{w}]=0,
$$

where $\delta_{1}$ and $\delta_{2}$ are calculated from

$$
\left\{\begin{aligned}
a_{2}\left(\delta_{1}^{2}+\delta_{2}^{2}\right) & =a_{2} a_{3}-a_{1}\left(2+a_{1}\right) \\
q^{2} a_{2} \delta_{1}^{2} \delta_{2}^{2} & =a_{1} \alpha^{2}+q^{2} a_{3}
\end{aligned}\right.
$$

In contrast, to the nature of the solution in the homogeneous case ( $\alpha=0)$, the solutions of (5.1) are not easily available due to the presence of the nonhomogeneity parameter $\alpha(\neq 0)$.

It is clear from (5.2) that the values of $\delta_{1}^{2}$ and $\delta_{2}^{2}$ will depend on the transform parameter $q$ and the nonhomogeneous parameter $\alpha$. It may be easily checked that $\delta_{1}^{2}$, $\delta_{2}^{2}$ will be real or complex according as

$$
\alpha^{2} \leq \text { or }>\frac{q^{2}\left[a_{2} a_{3}-a_{1}\left(2+a_{1}\right)\right]^{2}}{4 a_{1} a_{2}}-q^{2} \frac{a_{3}}{a_{1}} .
$$

Hence for a sufficiently large value of $\alpha, \delta_{1}^{2}$ and $\delta_{2}^{2}$ will assume complex values. Accordingly, separate attention is to be given to solving (5.1).

Since the solution of (5.1) depends on $\delta_{1}^{2}$ and $\delta_{2}^{2}$, henceforth we shall represent the solution corresponding to real $\delta_{1}^{2}$ and $\delta_{2}^{2}$ in part (a) and that corresponding to complex $\delta_{1}^{2}$ and $\delta_{2}^{2}$ in part (b) of any relevant equation.

Solutions of (5.1) compatible with the regularity condition are

$$
\begin{aligned}
& \left\{\begin{array}{l}
\bar{u}=A e^{-\lambda_{1} z}+B e^{-\lambda_{2} z} \\
\bar{w}=\frac{q\left\{\left(1+a_{1}\right) \lambda_{1}-\alpha a_{1}\right\}}{u_{1}-q^{2}} A e^{-\lambda_{1} z}+\frac{q\left\{\left(1+a_{1}\right) \lambda_{2}-\alpha a_{1}\right\}}{u_{2}-q^{2}} B e^{-\lambda_{2} z}
\end{array}\right. \\
& \left\{\begin{array}{l}
\bar{u}=e^{-\left(\alpha / 2+p_{1}\right) z}\left[A^{\prime} \cos \left(q_{1} z\right)+B^{\prime} \sin \left(q_{1} z\right)\right], \\
\bar{w}=e^{-\left(\alpha / 2+p_{1}\right) z}\left[\left(m_{2} B^{\prime}-n_{2} A^{\prime}\right) \cos \left(q_{1} z\right)-\left(n_{2} B^{\prime}+m_{2} A^{\prime}\right) \sin \left(q_{1} z\right)\right],
\end{array}\right.
\end{aligned}
$$

where $\lambda_{i}=\alpha / 2+\sqrt{\alpha^{2} / 4+q^{2} \delta_{i}^{2}}, u_{i}=a_{2} \lambda_{i}\left(\lambda_{i}-\alpha\right), i=1,2 ; p_{1}, q_{1}=\sqrt{\left(\xi_{1} \pm \xi_{2}\right) / 2}$, $\xi_{1}^{2}=\left(\alpha^{2} / 4+q^{2} \mu_{1}\right)^{2}+q^{4} \mu_{2}^{2}, \xi_{2}=\alpha^{2} / 4+q^{2} \mu_{1}, \mu_{1}=\left(a_{2} a_{3}-a_{1}\left(2+a_{1}\right)\right) / 2 a_{2}$, $\mu_{2}^{2}=\left(\alpha^{2} a_{1}+q^{2} a_{3}\right) /\left(a_{2} q^{2}\right)-\left(a_{2} a_{3}-a_{1}\left(2+a_{1}\right)\right)^{2} / 4 a_{2}^{2}, m_{2}=\left(t_{1} l_{1}-k_{1} m_{1}\right) /\left(k_{1}^{2}+t_{1}^{2}\right)$, $n_{2}=\left(k_{1} l_{1}+t_{1} m_{1}\right) /\left(k_{1}^{2}+t_{1}^{2}\right), t_{1}=\alpha a_{2} q_{1}-2 a_{2} q_{1}\left(\alpha / 2+p_{1}\right), l_{1}=\alpha a_{1} q-q(1+$ $\left.a_{1}\right)\left(\alpha / 2+p_{1}\right), k_{1}=a_{2}\left(\alpha / 2+p_{1}\right)^{2}-a_{2} q_{1}^{2}-\alpha a_{2}\left(\alpha / 2+p_{1}\right)-q^{2}, m_{1}=q q_{1}\left(1+a_{1}\right)$. 
Applying the transformed boundary conditions (4.5) the constants $A, B$ and $A^{\prime}, B^{\prime}$ are determined as

$$
A=A_{1} \bar{p} / q, \quad B=-A_{2} \bar{p} / q,
$$

where

$$
A_{1}=\frac{\left(\alpha-\lambda_{2}\right)\left(u_{1}-q^{2}\right)}{c_{44}^{0}\left(\lambda_{1}-\lambda_{2}\right)\left(a_{2} \lambda_{1}^{2}+a_{1} q^{2}\right)}, \quad A_{2}=\frac{\left(\alpha-\lambda_{1}\right)\left(u_{2}-q^{2}\right)}{c_{44}^{0}\left(\lambda_{1}-\lambda_{2}\right)\left(a_{2} \lambda_{2}^{2}+a_{1} q^{2}\right)}
$$

and

$$
A^{\prime}=(\bar{p} / q) M_{1}, \quad B^{\prime}=-(\bar{p} / q) M_{2},
$$

where $M_{1}=-q s_{4} / c_{44}^{0}\left(s_{1} s_{4}+s_{2} s_{3}\right), M_{2}=-q s_{3} / c_{44}^{0}\left(s_{1} s_{4}+s_{2} s_{3}\right), s_{1}=q a_{1}+$ $a_{2} n_{2}\left(\alpha / 2+p_{1}\right)+q_{1} m_{2} a_{2}, s_{2}=a_{2} m_{2}\left(\alpha / 2+p_{1}\right)+a_{2} q_{1} n_{2}, s_{3}=q n_{2}-\left(\alpha / 2+p_{1}\right)$, $s_{4}=q_{1}-q m_{2}$.

Hence from (5.3a), (5.3b) and (5.4a), (5.4b) we find that

$$
\bar{w}(q, 0)=R^{-1} \bar{p} / q,
$$

where

$$
\begin{aligned}
R= & c_{44}^{0}\left(a_{2} \lambda_{1}^{2}+a_{1} q^{2}\right)\left(a_{2} \lambda_{2}^{2}+a_{1} q^{2}\right) /\left[\lambda_{1} \lambda_{2}\left(\lambda_{1}+\lambda_{2}\right) q a_{2}\left(1+a_{1}\right)\right. \\
& \left.+\alpha a_{1} q^{3}+\alpha^{2} q a_{1} a_{2}\left(\lambda_{1}+\lambda_{2}\right)-\alpha q a_{2} \lambda_{1} \lambda_{2}-\alpha q a_{1} a_{2}\left(\lambda_{1}+\lambda_{2}\right)^{2}\right]
\end{aligned}
$$

and

$$
R=\frac{c_{44}^{0}}{q} \frac{s_{1} s_{4}+s_{2} s_{3}}{n_{2} s_{4}+m_{2} s_{3}}
$$

Taking the inverse Hankel transform of (5.5) and noting boundary condition (3.2), we can write

$$
D-f(r)=\int_{0}^{a} m p(m)\left[\int_{0}^{\infty} R^{-1} J_{0}(q r) J_{0}(q m) d q\right] d m
$$

In contrast to the homogeneous case in which $R$ given by (5.6a) is a pure constant, here $R$ is a function of $q$ and so the inversion of the integral in (5.7) for the function $p(r)$ is not easy. However, it is possible to reduce (5.7) to a Fredholm integral equation for the determination of $p(r)$.

Let us write

$$
C=\frac{c_{44}^{0}\left(a_{2} \gamma_{1}^{2}+a_{1}\right)\left(a_{2} \gamma_{2}^{2}+a_{1}\right)}{a_{2}\left(1+a_{1}\right)\left(\gamma_{1}+\gamma_{2}\right) \gamma_{1} \gamma_{2}},
$$

where $a_{2}\left(\gamma_{1}^{2}+\gamma_{2}^{2}\right)=a_{2} a_{3}-a_{1}\left(2+a_{1}\right), a_{2} \gamma_{1}^{2} \gamma_{2}^{2}=a_{3}$. 


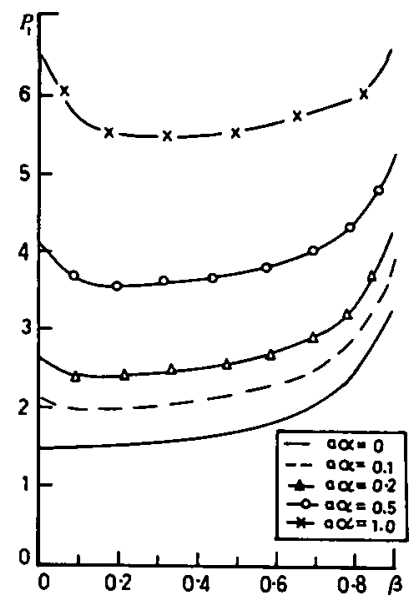

(a)

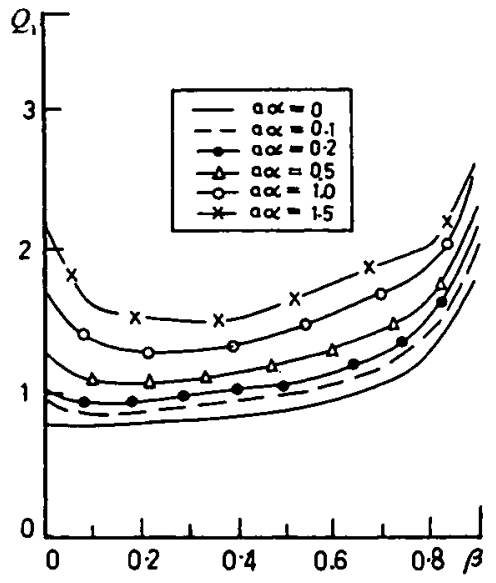

(b)

FIGURE 2. Variation of (a) $P_{1}$ and (b) $Q_{1}$ with $\beta(\eta=0)$.

Let

$$
J(r, m)=\int_{0}^{\infty} J_{0}(q r) J_{0}(q m) d q= \begin{cases}(2 / \pi m) K(r / m), & 0 \leq r \leq m ; \\ (2 / \pi r) K(m / r), & 0 \leq m \leq r\end{cases}
$$

and $T(r, m)=\int_{0}^{\infty}\left(R^{-1}-C^{-1}\right) J_{0}(q r) J_{0}(q m) d q$, where $K$ is the complete elliptic integral of the first kind.

Equation (5.7) can be written as

$$
D-f(r)=C^{-1} \int_{0}^{a} J(r, m) m p(m) d m+\int_{0}^{a} T(r, m) m p(m) d m .
$$

Let us multiply both sides of (5.8) by a factor $\xi / \sqrt{r^{2}-\xi^{2}}$ and integrate as follows:

$$
\begin{aligned}
\int_{0}^{r} \frac{\xi[D-f(\xi)] d \xi}{\sqrt{r^{2}-\xi^{2}}}= & C^{-1} \int_{0}^{r} \frac{\xi}{\sqrt{r^{2}-\xi^{2}}}\left[\int_{0}^{a} J(\xi, m) m p(m) d m\right] d \xi \\
& +\int_{0}^{r} \frac{\xi}{\sqrt{r^{2}-\xi^{2}}}\left[\int_{0}^{a} T(\xi, m) m p(m) d m\right] d \xi
\end{aligned}
$$

Interchanging the order of integration and using the result [4]

$$
\int_{0}^{r} \frac{\xi J_{0}(q \xi) d \xi}{\sqrt{r^{2}-\xi^{2}}}=\frac{\sin (r q)}{q}
$$

we get from (5.9) that

$$
\int_{0}^{r} \frac{\xi[D-f(\xi)] d \xi}{\sqrt{r^{2}-\xi^{2}}}=C^{-1} \int_{0}^{a} m p(m)\left[\int_{0}^{\infty} \frac{J_{0}(q m) \sin (r q)}{q} d q\right] d m
$$




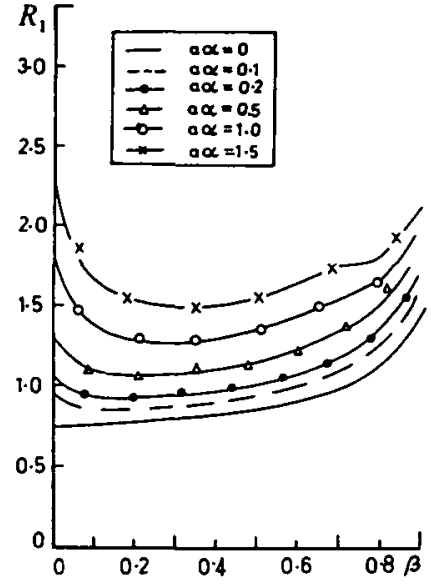

(a)

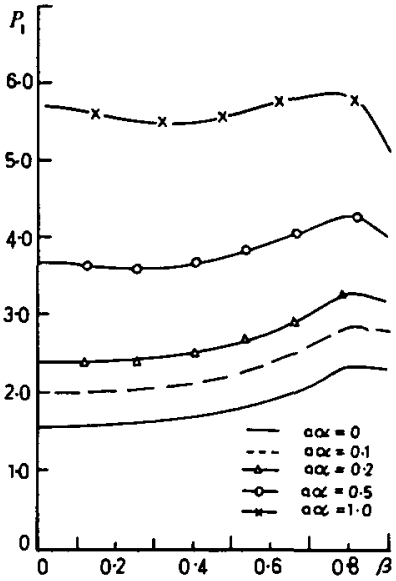

(b)

FIGURE 3. Variation of (a) $R_{1}$ with $\beta(\eta=0)$ and (b) $P_{1}$ with $\beta(\eta=0.2)$.

$$
+\int_{0}^{a} m p(m)\left[\int_{0}^{\infty} \frac{\left(R^{-1}-C^{-1}\right) J_{0}(q m) \sin (r q)}{q} d q\right] d m
$$

So we get

$$
\begin{aligned}
\frac{\partial}{\partial r} \int_{0}^{r} \frac{\xi[D-f(\xi)] d \xi}{\sqrt{r^{2}-\xi^{2}}} \\
=C^{-1} \int_{0}^{a} m p(m)\left[\int_{0}^{\infty} J_{0}(q m) \cos (r q) d q\right] d m \\
\quad+\int_{0}^{a} m p(m)\left[\int_{0}^{\infty}\left(R^{-1}-C^{-1}\right) J_{0}(q m) \cos (r q) d q\right] d m
\end{aligned}
$$

Multiplying (5.10) by a factor as in (5.9) and integrating with respect to $r$ between $\zeta$ and $a$, we get

$$
\begin{aligned}
\int_{\zeta}^{a} & \frac{r}{\sqrt{r^{2}-\zeta^{2}}}\left[\frac{\partial}{\partial r} \int_{0}^{r} \frac{\xi[D-f(\xi)] d \xi}{\sqrt{r^{2}-\xi^{2}}}\right] d r \\
= & \int_{\zeta}^{a} \frac{r}{\sqrt{r^{2}-\zeta^{2}}} C^{-1}\left[\int_{r}^{a} \frac{m p(m) d m}{\sqrt{m^{2}-r^{2}}}\right] d r \\
& +\int_{\zeta}^{a} \frac{r}{\sqrt{r^{2}-\zeta^{2}}}\left[\int_{0}^{a} m p(m)\left\{\int_{0}^{\infty}\left(R^{-1}-C^{-1}\right) J_{0}(q m) \cos (r q) d q\right\} d m\right] d r .
\end{aligned}
$$




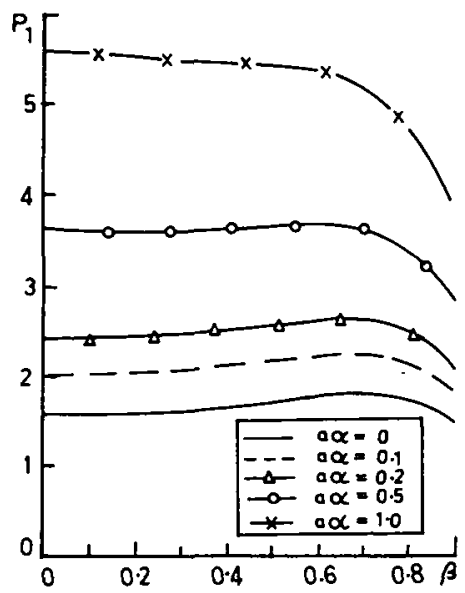

(a)

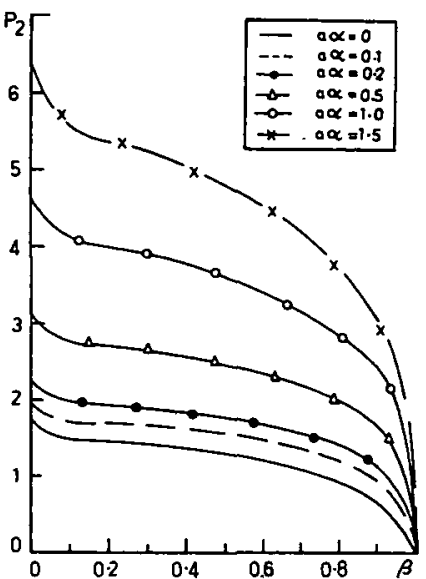

(b)

FIGURE 4. Variation of (a) $P_{1}$ with $\beta(\eta=0.5)$ and (b) $P_{2}$ with $\beta(\eta=0)$.

In the derivation of (5.11) we used the following result [4]:

$$
\int_{0}^{\infty} J_{0}(q m) \cos (q r) d q= \begin{cases}1 / \sqrt{m^{2}-r^{2}}, & m>r \\ 0, & m<r .\end{cases}
$$

Interchanging the integrals of the first part of the right-hand side and differentiating both sides of (5.11) we get

$$
\begin{aligned}
& \frac{\partial}{\partial r} \int_{r}^{a} \frac{\xi}{\sqrt{\xi^{2}-r^{2}}}\left[\frac{\partial}{\partial \xi} \int_{0}^{\xi} \frac{m(D-f(m))}{\sqrt{\xi^{2}-m^{2}}} d m\right] d \xi \\
& =-\frac{\pi}{2 C} r p(r) \\
& \quad+\frac{\partial}{\partial r} \int_{r}^{a} \frac{\xi}{\sqrt{\xi^{2}-r^{2}}}\left[\int_{0}^{a} t p(t) \int_{0}^{\infty}\left(R^{-1}-C^{-1}\right) J_{0}(q t) \cos (q \xi) d q d t\right] d \xi .
\end{aligned}
$$

Let us set $g(r)=D-f(r)$ and

$$
G(r)=\frac{\partial}{\partial r} \int_{0}^{r} \frac{m g(m) d m}{\sqrt{r^{2}-m^{2}}},
$$

then

$$
G(r)=g(0)+\int_{0}^{r} \frac{r g^{\prime}(m) d m}{\sqrt{r^{2}-m^{2}}} .
$$

Using (5.13), (5.12) may be written as

$$
\frac{2 C}{\pi}\left[\frac{G(a)}{\sqrt{a^{2}-r^{2}}}-\int_{r}^{a} \frac{G^{\prime}(\xi) d \xi}{\sqrt{\xi^{2}-r^{2}}}\right]
$$




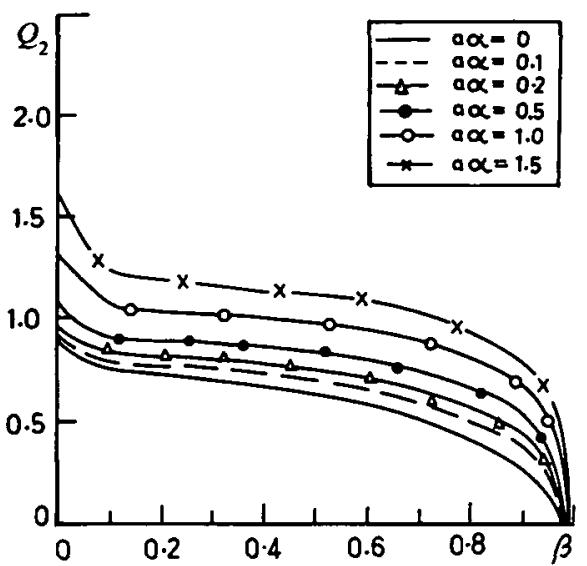

(a)

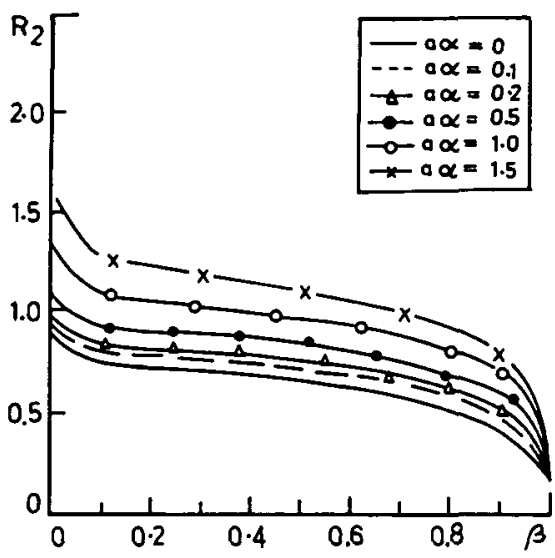

(b)

FIGURE 5. Variation of (a) $Q_{2}$ and (b) $R_{2}$ with $\beta(\eta=0)$.

$$
=p(r)-\frac{2}{\pi r} \frac{\partial}{\partial r} \int_{r}^{a} \frac{\xi}{\sqrt{\xi^{2}-r^{2}}}\left[\int_{0}^{a} t p(t) f_{1}(t, \xi) d t\right] d \xi
$$

where $f_{1}(t, \xi)=\int_{0}^{\infty}\left(C R^{-1}-1\right) J_{0}(q t) \cos (q \xi) d q$.

Equation (5.14) can now be put in the form

$$
p(r)+\int_{0}^{a} L(r, t) p(t) d t=\psi(r)
$$

where

$$
L(r, t)=\frac{2 t}{\pi}\left(\frac{f_{1}(t, a)}{\sqrt{a^{2}-r^{2}}}+\int_{r}^{a} \frac{1}{\sqrt{\xi^{2}-r^{2}}} \int_{0}^{\infty}\left(C R^{-1}-1\right) q J_{0}(q t) \sin (q \xi) d q d \xi\right)
$$

and

$$
\psi(r)=\frac{2 C}{\pi}\left[\frac{G(a)}{\sqrt{a^{2}-r^{2}}}-\int_{r}^{a} \frac{G^{\prime}(\xi) d \xi}{\sqrt{\xi^{2}-r^{2}}}\right]
$$

The Fredholm integral equation (5.15) will determine $p(r)$ for a known punch profile $f(r)$, consequently the transformed function $\bar{p}(q)$ is known from (4.6). Hence all the transformed functions corresponding to displacements and stresses are known and the problem reduces to that of finding the inverse Hankel transforms of those functions.

We can determine the penetration depth $D$ of the tip of a smooth profile from the condition $p(a)=0$ as follows. From (5.14), $p(a)=0$ implies $G(a)=0$, which gives after some calculations $D=\int_{0}^{a}\left(a f^{\prime}(r) / \sqrt{a^{2}-r^{2}}\right) d r$, which is the same as in the homogeneous case [8]. We note here that the smoothness condition is not satisfied 
in the case of a flat-ended cylindrical punch as pointed out in [9] and so the condition $p(a)=0$ cannot be used there.

The displacement components are given by

$$
\begin{aligned}
u(r, z)= & \int_{0}^{\infty} \bar{p}\left[A_{1} e^{-\lambda_{1} z}-A_{2} e^{-\lambda_{2} z}\right] J_{1}(q r) d q, \\
u(r, z)= & \int_{0}^{\infty} \bar{p} e^{-\left(\alpha / 2+p_{1}\right) z}\left[M_{1} \cos \left(q_{1} z\right)-M_{2} \sin \left(q_{1} z\right)\right] J_{1}(q r) d q, \\
w(r, z)= & \int_{0}^{\infty} q \bar{p}\left[\frac{A_{1}\left\{\left(1+a_{1}\right) \lambda_{1}-\alpha a_{1}\right\}}{u_{1}-q^{2}} e^{-\lambda_{1} z}\right. \\
& \left.-\frac{A_{2}\left\{\left(1+a_{1}\right) \lambda_{2}-\alpha a_{1}\right\}}{u_{2}-q^{2}} e^{-\lambda_{2} z}\right] J_{0}(q r) d q, \\
w(r, z)= & -\int_{0}^{\infty} \bar{p} e^{-\left(\alpha / 2+p_{1}\right) z}\left[\left(m_{2} M_{2}+n_{2} M_{1}\right) \cos \left(q_{1} z\right)\right. \\
& \left.+\left(m_{2} M_{1}-n_{2} M_{2}\right) \sin \left(q_{1} z\right)\right] J_{0}(q r) d q .
\end{aligned}
$$

The strain components are

$$
\begin{aligned}
\varepsilon_{r}+\varepsilon_{\theta}= & \int_{0}^{\infty} q \bar{p}\left[A_{1} e^{-\lambda_{1} z}-A_{2} e^{-\lambda_{2} z}\right] J_{0}(q r) d q \\
\varepsilon_{r}+\varepsilon_{\theta}= & \int_{0}^{\infty} q \bar{p} e^{-\left(\alpha / 2+p_{1}\right) z}\left[M_{1} \cos \left(q_{1} z\right)-M_{2} \sin \left(q_{1} z\right)\right] J_{0}(q r) d q \\
\varepsilon_{r}-\varepsilon_{\theta}= & -\int_{0}^{\infty} q \bar{p}\left[A_{1} e^{-\lambda_{1} z}-A_{2} e^{-\lambda_{2} z}\right] J_{2}(q r) d q \\
\varepsilon_{r}-\varepsilon_{\theta}= & -\int_{0}^{\infty} q \bar{p} e^{-\left(\alpha / 2+p_{1}\right) z}\left[M_{1} \cos \left(q_{1} z\right)-M_{2} \sin \left(q_{1} z\right)\right] J_{2}(q r) d q \\
\varepsilon_{z}= & -\int_{0}^{\infty} q \bar{p}\left[\frac{A_{1} \lambda_{1}\left\{\left(1+a_{1}\right) \lambda_{1}-\alpha a_{1}\right\}}{u_{1}-q^{2}} e^{-\lambda_{1} z}\right. \\
& \left.-\frac{A_{2} \lambda_{2}\left\{\left(1+a_{1}\right) \lambda_{2}-\alpha a_{1}\right\}}{u_{2}-q^{2}} e^{-\lambda_{2} z}\right] J_{0}(q r) d q \\
\varepsilon_{z}= & \int_{0}^{\infty} \bar{p} e^{-\left(\alpha / 2+p_{1}\right) z}\left[\left\{\left(\alpha / 2+p_{1}\right)\left(m_{2} M_{2}+n_{2} M_{1}\right)\right.\right. \\
& \left.-q_{1}\left(m_{2} M_{1}-n_{2} M_{2}\right)\right\} \cos \left(q_{1} z\right)+\left\{\left(\alpha / 2+p_{1}\right)\left(m_{2} M_{1}-n_{2} M_{2}\right)\right. \\
& \left.\left.+q_{1}\left(m_{2} M_{2}+n_{2} M_{1}\right)\right\} \sin \left(q_{1} z\right)\right] J_{0}(q r) d q \\
\varepsilon_{r z}= & -\int_{0}^{\infty} \frac{\bar{p}\left(\lambda_{1}-\alpha\right)\left(\lambda_{2}-\alpha\right)}{c_{44}^{0}\left(\lambda_{2}-\lambda_{1}\right)}\left[e^{-\lambda_{1} z}-e^{-\lambda_{2} z}\right] J_{1}(q r) d q \\
\varepsilon_{r z}= & \int_{0}^{\infty} \bar{p} e^{-\left(\alpha / 2+p_{1}\right) z}\left[\left(q\left(m_{2} M_{2}+n_{2} M_{1}\right)-M_{1}\left(\frac{\alpha}{2}+p_{1}\right)-M_{2} q_{1}\right) \cos \left(q_{1} z\right)\right. \\
& \left.+\left(q\left(m_{2} M_{1}-n_{2} M_{2}\right)+M_{2}\left(\frac{\alpha}{2}+p_{1}\right)-M_{1} q_{1}\right) \sin \left(q_{1} z\right)\right] J_{1}(q r) d q
\end{aligned}
$$


and the stress components are

$$
\begin{aligned}
\sigma_{r}+\sigma_{\theta}= & \int_{0}^{\infty} q \bar{p}\left[B_{1} e^{\left(\alpha-\lambda_{1}\right) z}-B_{2} e^{\left(\alpha-\lambda_{2}\right) z}\right] J_{0}(q r) d q \\
\sigma_{r}+\sigma_{\theta}= & c_{44}^{0} \int_{0}^{\infty} \bar{p} e^{\left(\alpha / 2-p_{1}\right) z}\left[\left(q\left(a_{3}+a_{4}\right) M_{1}+2 a_{1}\left(\alpha / 2+p_{1}\right)\left(m_{2} M_{2}+n_{2} M_{1}\right)\right.\right. \\
& \left.-2 a_{1} q_{1}\left(m_{2} M_{1}-n_{2} M_{2}\right)\right) \cos \left(q_{1} z\right) \\
& -\left(q\left(a_{3}+a_{4}\right) M_{2}-2 a_{1}\left(\alpha / 2+p_{1}\right)\left(m_{2} M_{1}-n_{2} M_{2}\right)\right. \\
& \left.\left.-2 a_{1} q_{1}\left(m_{2} M_{2}+n_{2} M_{1}\right)\right) \sin \left(q_{1} z\right)\right] J_{0}(q r) d q \\
\sigma_{r}-\sigma_{\theta}= & c_{44}^{0}\left(a_{4}-a_{3}\right) \int_{0}^{\infty} q \bar{p}\left[A_{1} e^{\left(\alpha-\lambda_{1}\right) z}-A_{2} e^{\left(\alpha-\lambda_{2}\right) z}\right] J_{2}(q r) d q \\
\sigma_{r}-\sigma_{\theta}= & c_{44}^{0}\left(a_{4}-a_{3}\right) \int_{0}^{\infty} q \bar{p} e^{\left(\alpha / 2-p_{1}\right) z}\left[M_{1} \cos \left(q_{1} z\right)-M_{2} \sin \left(q_{1} z\right)\right] J_{2}(q r) d q \\
\sigma_{z}= & -\int_{0}^{\infty} \frac{q \bar{p}}{\lambda_{2}-\lambda_{1}}\left[\left(\lambda_{2}-\alpha\right) e^{\left(\alpha-\lambda_{1}\right) z}-\left(\lambda_{1}-\alpha\right) e^{\left(\alpha-\lambda_{2}\right) z}\right] J_{0}(q r) d q \\
\sigma_{z}= & c_{44}^{0} \int_{0}^{\infty} e^{\left(\alpha / 2-p_{1}\right) z} \bar{p}\left[\left(q a_{1} M_{1}+a_{2}\left(\alpha / 2+p_{1}\right)\left(m_{2} M_{2}+n_{2} M_{1}\right)\right.\right. \\
& \left.-a_{2} q_{1}\left(m_{2} M_{1}-n_{2} M_{2}\right)\right) \cos \left(q_{1} z\right)-\left(q a_{1} M_{2}-a_{2}\left(\alpha / 2+p_{1}\right)\left(m_{2} M_{1}-n_{2} M_{2}\right)\right. \\
& \left.\left.-a_{2} q_{1}\left(m_{2} M_{2}+n_{2} M_{1}\right)\right) \sin \left(q_{1} z\right)\right] J_{0}(q r) d q
\end{aligned}
$$

where

$$
B_{i}=c_{44}^{0} a_{i}\left(\left(a_{3}+a_{4}\right)-2 a_{1} \lambda_{i} \frac{\left(1+a_{1}\right) \lambda_{i}-\alpha a_{1}}{u_{i}-q^{2}}\right) .
$$

\section{Special case: flat-ended cylindrical punch}

In the special case of a flat-ended cylindrical punch, $f(r)=0$ and hence we get

$$
p(r)+\int_{0}^{a} L(r, t) p(t) d t=\frac{2 C D}{\pi \sqrt{a^{2}-r^{2}}} .
$$

The contact force $F$ is then determined from $F=\int_{0}^{a} 2 \pi r p(r) d r$.

Finally, it may be checked that the results in the associated homogeneous medium as discussed by Pouyet and Lataillade [8] are easily obtainable by putting $\alpha=0$ in the corresponding results in our present article.

\section{Numerical results}

To assess the effects of non-homogeneity on stresses we first specify the nature of indentation $z=f(r)$. In our numerical works we shall consider two types of punches: 


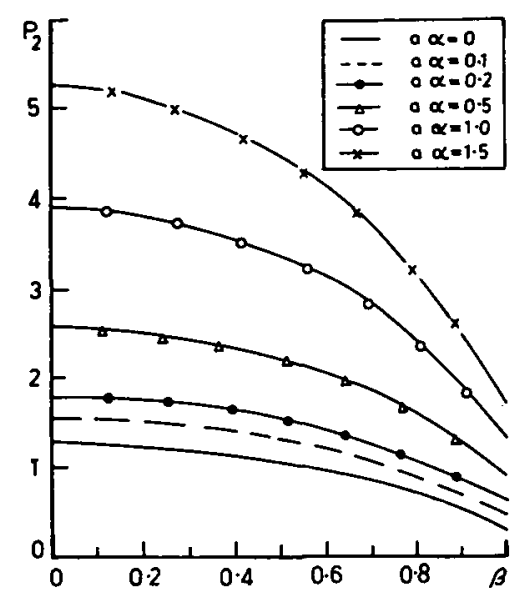

FIGURE 6. Variation of $P_{2}$ with $\beta(\eta=0.5)$.

(a) a flat-ended cylindrical punch for which $f(r)=0$ and (b) a paraboloidal punch for which $f(r)=r^{2} / 4 a$. Numerical computations are done taking various values of the non-homogeneity parameter $\alpha$ as mentioned in the figures. The values of the coefficients $a_{i}$ in (4.3) are taken for fibreglass epoxy composite material from [8]. The integral equation (5.15) is solved numerically to determine the pressure $p(r)$, which is subsequently used to determine the non-dimensional stress components.

For a flat-ended cylindrical punch we have shown variations of $P_{1}=-a \sigma_{z} /\left(D c_{44}^{0}\right)$, $Q_{1}=-a \sigma_{r} /\left(D c_{44}^{0}\right), R_{1}=-a \sigma_{\theta} /\left(D c_{44}^{0}\right)$ for different values of $\beta=r / a$ and $\eta=z / a$ in Figures 2-4 (a).

For the paraboloidal punch we have represented variations of $P_{2}=-\sigma_{z} / c_{44}^{0}$, $Q_{2}=-\sigma_{r} / c_{44}^{0}, R_{2}=-\sigma_{\theta} / c_{44}^{0}$ for different values of $\beta$ and $\eta$ in Figures 4 (b) -6 . The effects are quite clear from the figures.

\section{References}

[1] H. T. Danyluk, B. M. Singh and J. Vrbik, "The Reissner-Sagoci problem for a non-homogeneous half-space with a penny-shaped crack”, J. Engrg. Math. 29 (1995) 437-449.

[2] R. S. Dhaliwal and B. M. Singh, "The axisymmetric Boussinesq problem of an initially stressed Neo-Hookean half-space for a punch of arbitrary profile", Int. J. Engrg. Sci. 16 (1978) 379-385.

[3] G. M. L. Gladwell, Contact problems in the classical theory of elasticity (Sijthoff and Noordhoff, 1980).

[4] I. S. Gradshteyn and 1. M. Ryzhik, Table of integrals, series and products (Academic Press, New York, 1980) 682, 731.

[5] H. A. Z. Hassan and F. E. K. Rofaeel, "Reissner-Sagoci problem for a non-homogeneous half-space with surface constraint", J. Engrg. Math. 28 (1994) 117-127. 
[6] S. G. Lekhnitskii, Theory of elasticity of an anisotropic body (Mir, Moscow, 1981).

[7] M. Ozturk and F. Erdogan, "An axisymmetric crack in bonded materials with a nonhomogeneous interfacial zone under torsion", J. Appl. Mech. 62 (1995) 116-125.

[8] J. Pouyet and J. L. Lataillade, "Elastic strain of a transversely isotropic half-space caused by a rigid punch", Lett. Appl. Engrg. Sci. 17 (1979) 765-772.

[9] I. N. Sneddon, "The relation between load and penetration in the axisymmetric Boussinesq problem for a punch of arbitrary profile", Int. J. Engrg. Sci. 3 (1965) 47-57.

[10] D. A. Spence, "Self-similar solutions to adhesive contact problems with incremental loading", Proc. Roy. Soc. A 305 (1968) 55-80.

[11] D. A. Spence, "A Wiener-Hopf equation arising in elastic contact problems", Proc. Roy. Soc. A 305 (1968) 81-92.

[12] T. C. T. Ting, "The contact stresses between a rigid indenter and a viscoelastic half-space", J. Appl. Mech. 33 (1966) 845-854. 